TI 2011-127/1

Tinbergen Institute Discussion Paper

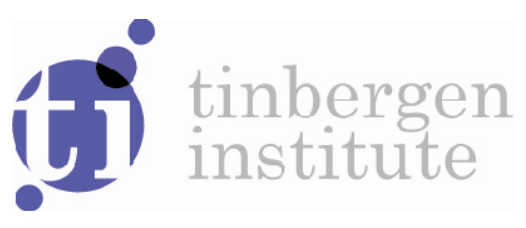

\title{
The Myerson Value and Superfluous Supports in Union Stable Systems
}

\author{
E. Algaba* \\ J.M. Bilbao" \\ R. van den Brink*** \\ J.J. López*
}

* Matemática Aplicada II, Escuela Superior de Ingenieros, Sevilla, Spain;

** Department of Econometrics, VU University, and Tinbergen Institute. 
Tinbergen Institute is the graduate school and research institute in economics of Erasmus University Rotterdam, the University of Amsterdam and VU University Amsterdam.

More TI discussion papers can be downloaded at http://www.tinbergen.nl

Tinbergen Institute has two locations:

Tinbergen Institute Amsterdam

Gustav Mahlerplein 117

1082 MS Amsterdam

The Netherlands

Tel.: +31(0)205251600

Tinbergen Institute Rotterdam

Burg. Oudlaan 50

3062 PA Rotterdam

The Netherlands

Tel.: +31(0)10 4088900

Fax: $+31(0) 104089031$

Duisenberg school of finance is a collaboration of the Dutch financial sector and universities, with the ambition to support innovative research and offer top quality academic education in core areas of finance.

DSF research papers can be downloaded at: http://www.dsf.nl/

Duisenberg school of finance

Gustav Mahlerplein 117

1082 MS Amsterdam

The Netherlands

Tel.: +31(0)20 5258579 


\title{
The Myerson value and superfluous supports in union stable systems
}

\author{
E. Algaba*, J. M. Bilbao*, R. van den Brink**, J. J. López* \\ *Matemática Aplicada II, Escuela Superior de Ingenieros, Camino de los \\ Descubrimientos, s/n, 41092 Sevilla, Spain (E-mail: ealgaba@us.es) \\ ** Department of Econometrics, VU University, De Boelelaan 1105, $1081 \mathrm{HV}$ \\ Amsterdam, The Netherlands (E-mail: jbrink@feweb.vu.nl)
}

\begin{abstract}
Cooperative games with partial cooperation cover a wider rank of real word situations than the classic model of cooperative games where every subset of a set of agents can form a coalition to execute the game. In this paper, the set of feasible coalitions which models the partial cooperation will be given by a union stable system. These systems contain, as particular cases, the communication situations and the permission structures, which are well-known both from a theoretical and applied point of view. Moreover, union stable systems are a natural framework for many other economic situations that arise in practice and which can not be modelled by these subsystems. In this paper, the goal is to make clear that there exists a close relationship between the Myerson value and the conference game. For that, we first analyze the relation between the restricted game and the conference game to establish later which effects a union stable system has on certain desirable properties of these games. Using the superfluous support property, defined through the conference game, new characterizations for the Myerson value are given in this context.
\end{abstract}

JEL Classification C71

Key words: Conference game, restricted game, union stable system, Myerson value, superfluous support property.

\section{Introduction}

In the classical model of transferable utility games it is generally assumed that any subset of a set $N$ of players can form a coalition and cooperate. However, many real world situations appear which require a more refined model which takes into account restrictions in cooperation. In Myerson's [14] model the feasible coalitions are induced by connected subgraphs. This line of research was continued by Owen [19], Borm et al. [7], van den Nouweland et al. [17] and Potters and Reijnierse [20]. However, as stated by Myerson himself, partial cooperation can not always be modelled by a graph, so, the original 
communication model has been generalized in several directions, for instance towards conference structures by Myerson [15], hypergraph communication situations by van den Nouweland et al. [17] and union stable systems by Algaba et al. [1, 2].

In Algaba et al. [1,2] it is assumed that if two feasible coalitions have common players, these ones will act as intermediaries between the two coalitions in order to establish meaningful communication in the union of these coalitions. These feasible coalition systems are called union stable systems. This mathematical feature is essential and the only requirement in these systems to establish the cooperation. In fact, different lines of research in the literature of cooperative games can be unified through these systems. For instance, the feasible coalitions coming from graph communication situations [14] and permission structures (see $[8,12]$ ) are special union stable systems. Furthermore, these systems have a close relation with the hypergraph communication situations [4]. Important properties on the class of union stable systems have been studied by Faigle et al. [11] who in this framework find a meaningful notion of supermodularity that extends Shapley's convex cooperative model.

The basis of a union stable system requires special attention and it is formed by its supports that in a communication situation are the edges of the graph and the singletons. In fact, the supports are those feasible coalitions that are not the union of two other non-disjoint feasible coalitions. All other feasible coalitions can be written as a union of non-disjoint supports. Two games that play an important role in games on union stable systems are the restricted game and the conference game, generalizing the corresponding games for communication situations. The restricted game is defined on the set of players and assigns to every coalition of players the worth that they can earn given the cooperation restrictions. The conference game is defined on the set of non unitary supports of the union stable system and assigns to every coalition of non unitary supports the worth that the 'grand coalition' consisting of all players can earn in the union stable cooperation structure generated by these supports. In this paper, we first analyze the relation between the restricted game and the conference game to establish later what effects a union stable system has on certain desirable properties of these games. In this way, relations between the properties of the restricted game and the conference game are investigated. We also consider the Myerson value, studied earlier in Algaba et al. [2], and show that from the study of the convexity of the conference game, we can provide sufficient conditions under which the Myerson value is in the core of the restricted game without requiring conditions on the structure or the restricted game. This emphasizes the role of the conference game on the Myerson value and a new approach to it.

After that we use supports and the conference game to provide axiomatizations of the Myerson value for games on union stable systems. In particular, we use the superfluous support property stating that the payoff allocation should not depend on supports that in some sense have no contribution. Together with component efficiency, component dummy, additivity and point 
unanimity we give a characterization of the Myerson value on a special class of union stable systems that contains those that arise from cycle-free graphs (and on which the position value was characterized in [1]). We also provide an axiomatization of the Myerson value on the class of all union stable systems by replacing the superfluous support property by the new strong superfluous support property which is defined using the restricted game, and inspired by the corresponding axiom for communication situations in van den Nouweland [18].

The paper is organized as follows. Section 2 recalls the general concepts on classical cooperation, the main definitions on restricted cooperation by means of union stable systems including the crucial driving notions of basis and supports, the restricted game, the conference game as well as the Myerson value and the position value. In Section 3, we analyze the relation between the restricted game and the conference game and the inheritance of properties. Section 4 contains axiomatizations of the Myerson value. We first provide an axiomatization for games on the special class of union stable systems that generalize the set of connected coalitions in a cycle-free graph, using the superfluous support property. Second, we provide an axiomatization on the class of all union stable systems by means of the strong superfluous support property. Finally, a section of conclusions is given.

\section{Preliminaries}

\subsection{Cooperative TU-games}

A cooperative transferable utility (TU)-game is a pair $(N, v)$ where $N=$ $\{1, \ldots, n\}$ is a finite set of players and $v: 2^{N} \rightarrow \mathbb{R}$ with $v(\emptyset)=0$, is a characteristic function. A game $(N, v)$ is non-negative if $v(S) \geq 0$ for all $S \subseteq N$.

A distribution of the amount $v(N)$ among the players will be represented by a real-valued vector $x \in \mathbb{R}^{n}$. Here $x_{i}$ represents the payoff to player $i$ according to the involved payoff vector $x$. A solution is a real-valued function that assigns a payoff vector to every game $v$. A solution $f$ satisfies the efficiency principle if $\sum_{j \in N} f_{j}(v)=v(N)$.

The most well-known solution concept is the Shapley value (Shapley [21]) given by

$$
\Phi_{i}(v)=\sum_{S \subseteq N \backslash\{i\}} \frac{(n-|S|-1) !(|S|) !}{n !}(v(S \cup\{i\})-v(S)) \text {, for all } i \in N .
$$

The core of a game $(N, v)$ is the set

$$
C(v)=\left\{x \in \mathbb{R}^{n}: x(N)=v(N), x(S) \geq v(S), \text { for all } S \subset N\right\},
$$

where $x(S)=\sum_{i \in S} x_{i}$ and $x(\emptyset)=0$.

Bondareva [6] and Shapley [22] state that a game $(N, v)$ is balanced if and only if it has a nonempty core. A game is called totally balanced if each subgame is balanced. 
A game $(N, v)$ is superadditive if cooperation is profitable, i.e.,

$$
v(S \cup T) \geq v(S)+v(T) \text { for all disjoint coalitions } S, T \in 2^{N} .
$$

A game $(N, v)$ is convex if

$$
v(S \cup T)+v(S \cap T) \geq v(S)+v(T) \text { for all coalitions } S, T \in 2^{N} .
$$

\subsection{Union stable systems}

Let $N=\{1, \ldots, n\}$ be a finite set of players and $\mathcal{F} \subseteq 2^{N}$ a set system of feasible coalitions. The set system $\mathcal{F}$ is called union stable if for all $A, B \in \mathcal{F}$ with $A \cap B \neq \emptyset$ it is satisfied that $A \cup B \in \mathcal{F}$.

Many real world situations find their natural framework in these structures. For instance, suppose that player 1 is a homeowner who wants to sell his/her house. Player 1 has signed a contract with a real estate agent that represents player 2 . So, player 1 only can sell his/her house by means of player 2. There are two buyers, players 3 and 4 . Notice that in this economic application, the family of feasible coalitions that can generate a surplus are only those which make possible that the seller can sell his/her house. Therefore, the coalitions which can trade are

$$
\mathcal{F}=\{\{1,2,3\},\{1,2,4\},\{1,2,3,4\}\} .
$$

An important subclass of union stable systems is communication situations as considered in Myerson [14]. A communication situation is a triple $(N, v, E)$, where $(N, v)$ is a game and $(N, E)$ is a simple graph. It is easy to see that the set system $\mathcal{F}$, defined by those coalitions which induce connected subgraphs is a union stable system. However, in practice, a union stable system can not always be modelled by a communication situation (see van den Brink [9] for a characterization of the set systems that can be obtained as connected coalitions in a communication graph). For example, the set system $\mathcal{F}$ pointed out above with one seller/two buyers and a real state agent as intermediary is a union stable system which cannot be the set of connected coalitions in a communication graph. So, union stable systems not only allow for a generalization of set systems derived from communication graphs but also a better understanding and insight of them and their applications.

Let $\mathcal{F}$ be a union stable system and $\mathcal{G} \subseteq \mathcal{F}$. The following families are defined inductively

$$
\mathcal{G}^{(0)}=\mathcal{G}, \quad \mathcal{G}^{(n)}=\left\{S \cup T: S, T \in \mathcal{G}^{(n-1)}, S \cap T \neq \emptyset\right\} \quad(n=1,2, \ldots)
$$

Notice that $\mathcal{G}^{(0)} \subseteq \mathcal{G}^{(n-1)} \subseteq \mathcal{G}^{(n)} \subseteq \mathcal{F}$, since $\mathcal{G} \subseteq \mathcal{F}$ and $\mathcal{F}$ is union stable. We define $\overline{\mathcal{G}}$ by $\overline{\mathcal{G}}=\mathcal{G}^{(\bar{k})}$, where $k$ is the smallest integer such that $\mathcal{G}^{(k+1)}=\mathcal{G}^{(k)}$.

For each union stable family $\mathcal{F}$, it is interesting to find a minimal subset $\mathcal{B}(\mathcal{F})$ such that $\overline{B(\mathcal{F})}=\mathcal{F}$. So, the following set is well-defined:

$$
\mathcal{E}(\mathcal{F})=\{G \in \mathcal{F}: G=A \cup B, A \neq G, B \neq G, A, B \in \mathcal{F}, A \cap B \neq \emptyset\} .
$$


The set $\mathcal{B}(\mathcal{F})=\mathcal{F} \backslash \mathcal{E}(\mathcal{F})$, is called the basis of $\mathcal{F}$, and the elements of $\mathcal{B}(\mathcal{F})$ are called supports of $\mathcal{F}$. We remark that the basis $B(\mathcal{F})$ is the minimal subset of the union stable system $\mathcal{F}$ such that $\overline{B(\mathcal{F})}=\mathcal{F}$ (see Algaba et al. $[1])$.

Let $\mathcal{G} \subseteq 2^{N}$ be a set system and let $S \subseteq N$. A set $T \subseteq S$ is called a $\mathcal{G}$-component of $S$ if $T \in \mathcal{G}$ and there exists no $T^{\prime} \in \mathcal{G}$ such that $T \subset T^{\prime} \subseteq S$. Therefore, the $\mathcal{G}$-components of $S$ are the maximal feasible coalitions that belong to $\mathcal{G}$ and are contained in $S$. We denote by $C_{\mathcal{G}}(S)$ the collection of the $\mathcal{G}$-components of $S$. Union stable systems can be characterized in terms of the $\mathcal{F}$-components of a coalition in the following way: The set system $\mathcal{F} \subseteq 2^{N}$ is union stable if and only if for any $S \subseteq N$ with $C_{\mathcal{F}}(S) \neq \emptyset$, the $\mathcal{F}$-components of $S$ are a collection of pairwise disjoint subsets of $S$.

Let $(N, v)$ be a cooperative game and $\mathcal{F} \subseteq 2^{N}$ a union stable system. Let $\mathcal{B}(\mathcal{F})$ be the basis of $\mathcal{F}$ and $\mathcal{C}(\mathcal{F})=\{B \in \mathcal{B}(\mathcal{F}):|B| \geq 2\}$. If there is no confusion we will just write $\mathcal{B}$ and $\mathcal{C}$. The $\mathcal{F}$-restricted game $v^{\mathcal{F}}: 2^{N} \rightarrow \mathbb{R}$, is defined on the player set and is given by $v^{\mathcal{F}}(S)=\sum_{T \in C_{\mathcal{F}}(S)} v(T)$. On the other hand, the conference game is defined on the basis of a union stable system and it is the game $\left(\mathcal{C}, v^{\mathcal{C}}\right)$ where $v^{\mathcal{C}}: 2^{\mathcal{C}} \rightarrow \mathbb{R}$, is given by $v^{\mathcal{C}}(\mathcal{A})=$ $v^{\overline{\mathcal{A}}}(N)$.

Note that the game $\left(\mathcal{C}, v^{\mathcal{C}}\right)$ is well defined since for each $\mathcal{A} \subseteq \mathcal{C}, \overline{\mathcal{A}}$ is a union stable system. The $\mathcal{F}$-restricted game focuses on the role of a player in creating economic possibilities and establishing meaningful communication among the players whereas the conference game measures the economic value of the grand coalition when specific parts of the cooperation structure are considered.

The two above definitions extend the point game (introduced by Myerson [14]) and the arc game (introduced by Borm et al. [7]) for communication situations, where for a communication situation $(N, v, E)$ we have that $\mathcal{C}=$ $\{\{i, j\}:\{i, j\} \in E\}$.

A union stable cooperation structure is a triple $(N, v, \mathcal{F})$ where $N=$ $\{1, \ldots, n\}$ is the set of players, $(N, v)$ is a game with $v: 2^{N} \rightarrow \mathbb{R}$ satisfying $v(\emptyset)=0$, and $\mathcal{F}$ is a union stable system. For convenience, we assume from now on that the underlying game $(N, v)$ is zero-normalized.

The set of all union stable cooperation structures on $N$ will be denoted by $U S^{N}=\{(N, v, \mathcal{F}): \mathcal{F}$ is union stable $\}$.

We will denote by $U S I^{N}$ a special subclass of $U S^{N}$ where the following two conditions are satisfied:

(1) For all $S, T \in \mathcal{F}$, with $|S \cap T| \geq 2$ we have $S \cap T \in \mathcal{F}$.

(2) All non-unitary feasible coalitions can be written in a unique way as a union of non-unitary supports.

Notice that this subclass of union stable cooperation structures generalizes those communication situations for which the graphs do not contain cycles. 


\subsection{Allocation rules for union stable structures}

An allocation rule on $U S^{N}$ is a map $\gamma$ that assigns to each union stable cooperation structure $(N, v, \mathcal{F})$ a payoff vector, $\gamma(N, v, \mathcal{F}) \in \mathbb{R}^{n}$.

Both the Myerson value and the position value are defined from the Shapley value $[21]$ of the two games that were defined above, the $\mathcal{F}$-restricted game and the conference game respectively. The Myerson value was introduced in Myerson [14] and later extended in [15]. Myerson pointed out the need to generalize this model towards restricted cooperation situations which can not be modelled by a graph. This idea has been studied by van den Nouweland et al. [17] and Algaba et al. [2]. So, given $(N, v, \mathcal{F})$ a union stable cooperation structure, the Myerson value denoted by $\mu(N, v, \mathcal{F}) \in \mathbb{R}^{n}$ is defined by

$$
\mu(N, v, \mathcal{F})=\Phi\left(N, v^{\mathcal{F}}\right) .
$$

The position value for graph communication situations was first introduced in Meesen [13] and studied in Borm et al. [7]. This value was extended to hypergraph communication situations in [17] and is defined in union stable cooperation structures in [1]. Let $(N, v, \mathcal{F})$ be a union stable cooperation structure. The position value, denoted by $\pi(N, v, \mathcal{F}) \in \mathbb{R}^{n}$, is given by

$$
\pi_{i}(N, v, \mathcal{F})=\sum_{C \in \mathcal{C}_{i}(\mathcal{F})} \frac{1}{|C|} \Phi_{C}\left(\mathcal{C}, v^{\mathcal{C}}\right), \text { for } i \in N
$$

where $\mathcal{C}_{i}(\mathcal{F})=\{C \in \mathcal{C}: i \in C\}$. When there is no confusion we will often write $\mathcal{C}_{i}$ instead of $\mathcal{C}_{i}(\mathcal{F})$.

The following example illustrates the concepts of Myerson and position values for the economic application introduced in (1).

Consider the player set $N=\{1,2,3,4\}$ and the union stable family given by $\mathcal{F}=\{\{1,2,3\},\{1,2,4\}, N\}$. Let $v: 2^{N} \rightarrow \mathbb{R}$ be defined by $v(S)=|S|-1$ if $|S| \geq 1$. Then,

$$
\mathcal{B}=\mathcal{C}=\{\{1,2,3\},\{1,2,4\}\}
$$

In this case,

$$
v^{\mathcal{F}}(S)= \begin{cases}|S|-1, & \text { if } S \in \mathcal{F} \\ 0, & \text { otherwise }\end{cases}
$$

Hence, the Myerson value for this situation is

$$
\mu(N, v, \mathcal{F})=\left(\frac{13}{12}, \frac{13}{12}, \frac{5}{12}, \frac{5}{12}\right) .
$$

The conference game is given by

\begin{tabular}{||c|c|c|c||}
\hline $\mathcal{A} \subseteq \mathcal{C}$ & $\overline{\mathcal{A}}$ & $C_{\overline{\mathcal{A}}}(N)$ & $v^{\mathcal{C}}(\mathcal{A})$ \\
\hline \hline$\{\{1,2,3\}\}$ & $\{\{1,2,3\}\}$ & $\{\{1,2,3\}\}$ & 2 \\
\hline$\{\{1,2,4\}\}$ & $\{\{1,2,4\}\}$ & $\{\{1,2,4\}\}$ & 2 \\
\hline$\{\{1,2,3\},\{1,2,4\}\}$ & $\{\{1,2,3\},\{1,2,4\},\{1,2,3,4\}\}$ & $\{\{1,2,3,4\}\}$ & 3 \\
\hline
\end{tabular}


Thus,

$$
\pi(N, v, \mathcal{F})=\left(1,1, \frac{1}{2}, \frac{1}{2}\right) .
$$

\section{Relation between the conference game and the restricted game}

In the same way that there exists a connection between the arc game and the point game as shown in van den Nouweland and Borm [16], here we establish a relation between the restricted game $\left(N, v^{\mathcal{F}}\right)$, and the conference game $\left(\mathcal{C}, v^{\mathcal{C}}\right)$. Notice that the Myerson value and the position value have been defined from the Shapley value of the restricted game and the conference game respectively. So, the relationship between the restricted game and the conference game as well as the inheritance of properties as superadditivity, balancedness and convexity allows for a deeper knowledge and better insight into them.

Theorem 1 Let $(N, v, \mathcal{F})$ be a union stable cooperation structure and $\left(\mathcal{C}, v^{\mathcal{C}}\right)$ the associated conference game. Then, for each $S \subseteq N$ it is satisfied that

$$
v^{\mathcal{F}}(S)=v^{\mathcal{C}}\left(\mathcal{C}_{S}\right)
$$

where $\mathcal{C}_{S}=\{C \in \mathcal{C}: C \subseteq S\}$

Proof. Let $S \subseteq N$. If $C_{\mathcal{F}}(S)=\emptyset$, it is straightforward that $\mathcal{C}_{S}=\emptyset$ and thus $v^{\mathcal{F}}(S)=0$. Otherwise, $v^{\mathcal{F}}(S)=\sum_{T \in C_{\mathcal{F}}(S)} v(T)$. Consider $\mathcal{F}_{S}=\{F \in \mathcal{F}$ : $F \subseteq S\}$. Then $\mathcal{F}_{S}$ is a union stable system such that $C_{\mathcal{F}_{S}}(N)=C_{\mathcal{F}}(S)$. Its basis is $\mathcal{B}_{S}=\{B \in \mathcal{B}: B \subseteq S\}$. Let $\mathcal{C}_{S}$ be the set formed by the supports of $\mathcal{B}_{S}$ which have cardinal at least two. It holds that $\mathcal{C}_{S} \subseteq \mathcal{C}$. Therefore, for any coalition $S \subseteq N$ it is satisfied that

$$
v^{\mathcal{C}}\left(\mathcal{C}_{S}\right)=v^{\overline{\mathcal{C}}_{S}}(N)=v^{\overline{\mathcal{B}}_{S}}(N)=\sum_{M \in C_{\overline{\mathcal{B}}_{S}}(N)} v(M)=\sum_{T \in C_{\mathcal{F}}(S)} v(T),
$$

since $C_{\mathcal{F}_{S}}(N)=C_{\mathcal{F}}(S)$ and the game $(N, v)$ is zero-normalized.

The following results state the inheritance of balancedness, superadditivity and convexity from the conference game to the restricted game. First, we consider balancedness.

Theorem 2 Let $(N, v, \mathcal{F})$ be a union stable cooperation structure. If $\left(\mathcal{C}, v^{\mathcal{C}}\right)$ is non-negative and balanced, then $\left(N, v^{\mathcal{F}}\right)$ is balanced.

Proof. As $\left(\mathcal{C}, v^{\mathcal{C}}\right)$ is non-negative and balanced,

$$
C\left(v^{\mathcal{C}}\right)=\left\{y \in \mathbb{R}_{+}^{|\mathcal{C}|}: \sum_{C \in \mathcal{C}} y_{C}=v^{\mathcal{C}}(\mathcal{C}), \sum_{A \in \mathcal{A}} y_{A} \geq v^{\mathcal{C}}(\mathcal{A}), \forall \mathcal{A} \subseteq \mathcal{C}\right\} \neq \emptyset .
$$


Let $y \in C\left(v^{\mathcal{C}}\right)$. From $y$, we construct the vector $x \in \mathbb{R}^{n}$ in the following way

$$
x_{i}= \begin{cases}\sum_{C \in \mathcal{C}_{i}} \frac{1}{|C|} y_{C}, & \text { if } \mathcal{C}_{i} \neq \emptyset \\ 0, & \text { otherwise }\end{cases}
$$

for each $i \in N$. We have to prove that $x \in C\left(v^{\mathcal{F}}\right)$, i.e.,

$$
\sum_{i \in N} x_{i}=v^{\mathcal{F}}(N) \text { and } \sum_{i \in S} x_{i} \geq v^{\mathcal{F}}(S), \forall S \subset N
$$

Indeed, first

$$
\begin{aligned}
\sum_{i \in N} x_{i} & =\sum_{i \in N}\left[\sum_{C \in \mathcal{C}_{i}} \frac{1}{|C|} y_{C}\right]=\sum_{C \in \mathcal{C}}\left[\sum_{i \in C} \frac{1}{|C|} y_{C}\right] \\
& =\sum_{C \in \mathcal{C}}\left[|C| \frac{1}{|C|} y_{C}\right]=\sum_{C \in \mathcal{C}} y_{C}=v^{\mathcal{C}}(\mathcal{C}) . \\
& =v^{\mathcal{F}}(N) .
\end{aligned}
$$

On the other hand,

$$
\sum_{i \in S} x_{i}=\sum_{i \in S}\left[\sum_{C \in \mathcal{C}_{i}} \frac{1}{|C|} y_{C}\right] .
$$

By Theorem 1, for each $S \subset N, v^{\mathcal{C}}\left(\mathcal{C}_{S}\right)=v^{\mathcal{F}}(S)$ and as $y_{C} \geq 0$ and moreover $\left\{C \in \mathcal{C}_{S}: i \in C\right\} \subseteq \mathcal{C}_{i}$, we have

$$
\begin{aligned}
\sum_{i \in S} x_{i} & =\sum_{i \in S}\left[\sum_{C \in \mathcal{C}_{i}} \frac{1}{|C|} y_{C}\right] \geq \sum_{i \in S}\left[\sum_{\left\{C \in \mathcal{C}_{S}: i \in C\right\}} \frac{1}{|C|} y_{C}\right] \\
& =\sum_{C \in \mathcal{C}_{S}}\left[\frac{1}{|C|}|C|\right] y_{C}=\sum_{C \in \mathcal{C}_{S}} y_{C} \geq v^{\mathcal{C}}\left(\mathcal{C}_{S}\right)=v^{\mathcal{F}}(S),
\end{aligned}
$$

where the last inequality holds because $y \in C\left(v^{\mathcal{C}}\right)$. Hence we conclude that $\left(N, v^{\mathcal{F}}\right)$ is balanced.

A similar result holds with respect to superadditivity.

Theorem 3 Let $(N, v, \mathcal{F})$ be a union stable cooperation structure. If $\left(\mathcal{C}, v^{\mathcal{C}}\right)$ is superadditive and non-negative, then $\left(N, v^{\mathcal{F}}\right)$ is superadditive.

Proof. Let $S, T \subseteq N, S \cap T=\emptyset$. We have to prove that

$$
v^{\mathcal{F}}(S \cup T) \geq v^{\mathcal{F}}(S)+v^{\mathcal{F}}(T)
$$

By Theorem 1,

$$
v^{\mathcal{F}}(S)=v^{\mathcal{C}}\left(\mathcal{C}_{S}\right), \quad v^{\mathcal{F}}(T)=v^{\mathcal{C}}\left(\mathcal{C}_{T}\right), \quad v^{\mathcal{F}}(S \cup T)=v^{\mathcal{C}}\left(\mathcal{C}_{S \cup T}\right),
$$


therefore, the expression (2) is equivalent to

$$
v^{\mathcal{C}}\left(\mathcal{C}_{S \cup T}\right) \geq v^{\mathcal{C}}\left(\mathcal{C}_{S}\right)+v^{\mathcal{C}}\left(\mathcal{C}_{T}\right) .
$$

For that, it suffices to prove that $\mathcal{C}_{S} \cap \mathcal{C}_{T}=\emptyset$ and that $\mathcal{C}_{S} \cup \mathcal{C}_{T} \subseteq \mathcal{C}_{S \cup T}$ since, by the superadditivity and the monotonicity of the game $\left(\mathcal{C}, v^{\mathcal{C}}\right)$, we have

$$
v^{\mathcal{C}}\left(\mathcal{C}_{S}\right)+v^{\mathcal{C}}\left(\mathcal{C}_{T}\right) \leq v^{\mathcal{C}}\left(\mathcal{C}_{S} \cup \mathcal{C}_{T}\right) \leq v^{\mathcal{C}}\left(\mathcal{C}_{S \cup T}\right) .
$$

On the one hand, since

$$
\mathcal{C}_{S} \subseteq 2^{S}, \quad \mathcal{C}_{T} \subseteq 2^{T}, \quad S \cap T=\emptyset \text { we have } \mathcal{C}_{S} \cap \mathcal{C}_{T}=\emptyset .
$$

On the other hand, by construction of $\mathcal{C}_{S}, \mathcal{C}_{T}$ and $\mathcal{C}_{S \cup T}$ we conclude that $\mathcal{C}_{S} \cup \mathcal{C}_{T} \subseteq \mathcal{C}_{S \cup T}$

The next example illustrates that, in general, balancedness or superadditivity of the underlying restricted game does not imply this property for the conference game.

Example 1 Let $(N, v, \mathcal{F})$ be a union stable cooperation structure, where $(N, \mathcal{F})$ is the union stable system considered in (1), i.e., $N=\{1,2,3,4\}$, the feasible coalition system is $\mathcal{F}=\{\{1,2,3\},\{1,2,4\},\{1,2,3,4\}\}$ and let the game be given by

$$
v(S)= \begin{cases}|S|, & \text { if }|S| \geq 2, \\ 0, & \text { otherwise. }\end{cases}
$$

Note, that game $(N, v)$ is totally balanced and therefore so is the game $\left(N, v^{\mathcal{F}}\right)$ (see [3]). The corresponding conference game $\left(\mathcal{C}, v^{\mathcal{C}}\right)$ is given by $v^{\mathcal{C}}(\{\{1,2,3\}\})=$ $v^{\mathcal{C}}(\{\{1,2,4\}\})=3$ and $v^{\mathcal{C}}(\{\{1,2,3\},\{1,2,4\}\})=4$, and thus is not balanced, since $\mathcal{C}=\mathcal{B}=\{\{1,2,3\},\{1,2,4\}\}$ and hence,

$$
C\left(v^{\mathcal{C}}\right)=\left\{y \in \mathbb{R}^{|\mathcal{C}|}: y_{\{1,2,3\}}+y_{\{1,2,4\}}=4, y_{\{1,2,3\}} \geq 3, y_{\{1,2,4\}} \geq 3\right\}=\emptyset .
$$

The game $(N, v)$ is also superadditive and it is easy to show that then so is the game $\left(N, v^{\mathcal{F}}\right)$. However, the conference game $\left(\mathcal{C}, v^{\mathcal{C}}\right)$ is not superadditive, since

$$
v^{\mathcal{C}}(\{\{1,2,3\},\{1,2,4\}\}) \nsupseteq v^{\mathcal{C}}(\{\{1,2,3\}\})+v^{\mathcal{C}}(\{\{1,2,4\}\}),
$$

As mentioned above this example can not be modelled by a communication situation.

Next, we study the transmission of convexity from the game $\left(\mathcal{C}, v^{\mathcal{C}}\right)$ to $\left(N, v^{\mathcal{F}}\right)$.

Theorem 4 Let $(N, v, \mathcal{F})$ be a union stable cooperation structure. If the conference game $\left(\mathcal{C}, v^{\mathcal{C}}\right)$ is non-negative and convex then $\left(N, v^{\mathcal{F}}\right)$ is convex. 
Proof. Let $i \in N$ and $S, T$ such that $S \subseteq T \subseteq N \backslash\{i\}$. We have to prove that

$$
v^{\mathcal{F}}(S \cup\{i\})-v^{\mathcal{F}}(S) \leq v^{\mathcal{F}}(T \cup\{i\})-v^{\mathcal{F}}(T) .
$$

By Theorem 1, it holds that

$$
\begin{array}{lll}
v^{\mathcal{F}}(S \cup\{i\})=v^{\mathcal{C}}\left(\mathcal{C}_{S \cup\{i\}}\right), & & v^{\mathcal{F}}(S)=v^{\mathcal{C}}\left(\mathcal{C}_{S}\right), \\
v^{\mathcal{F}}(T \cup\{i\})=v^{\mathcal{C}}\left(\mathcal{C}_{T \cup\{i\}}\right), & v^{\mathcal{F}}(T)=v^{\mathcal{C}}\left(\mathcal{C}_{T}\right) .
\end{array}
$$

Therefore, the above is equivalent to prove that

$$
v^{\mathcal{C}}\left(\mathcal{C}_{S \cup\{i\}}\right)-v^{\mathcal{C}}\left(\mathcal{C}_{S}\right) \leq v^{\mathcal{C}}\left(\mathcal{C}_{T \cup\{i\}}\right)-v^{\mathcal{C}}\left(\mathcal{C}_{T}\right) .
$$

On the one hand, as $\mathcal{C}_{S \cup\{i\}}, \mathcal{C}_{T \cup\{i\}}, \mathcal{C}_{S}$ and $\mathcal{C}_{T}$ are the sets of non-unitary supports of the union stable systems $\left(N, \mathcal{F}_{S \cup\{i\}}\right),\left(N, \mathcal{F}_{T \cup\{i\}}\right),\left(N, \mathcal{F}_{S}\right)$ and $\left(N, \mathcal{F}_{T}\right)$, respectively, it is satisfied that

$$
\mathcal{C}_{S \cup\{i\}} \cup \mathcal{C}_{T} \subseteq \mathcal{C}_{T \cup\{i\}} \text { and } \mathcal{C}_{S \cup\{i\}} \cap \mathcal{C}_{T}=\mathcal{C}_{S}
$$

On the other hand, as $\left(\mathcal{C}, v^{\mathcal{C}}\right)$ is non negative and superadditive, we have

$$
v^{\mathcal{C}}\left(\mathcal{C}_{T \cup\{i\}}\right) \geq v^{\mathcal{C}}\left(\mathcal{C}_{S \cup\{i\}} \cup \mathcal{C}_{T}\right) .
$$

Moreover, by convexity of the game $\left(\mathcal{C}, v^{\mathcal{C}}\right)$ it holds

$$
\begin{aligned}
v^{\mathcal{C}}\left(\mathcal{C}_{S \cup\{i\}} \cup \mathcal{C}_{T}\right)-v^{\mathcal{C}}\left(\mathcal{C}_{T}\right) & \geq v^{\mathcal{C}}\left(\mathcal{C}_{S \cup\{i\}}\right)-v^{\mathcal{C}}\left(\mathcal{C}_{S \cup\{i\}} \cap \mathcal{C}_{T}\right) \\
& =v^{\mathcal{C}}\left(\mathcal{C}_{S \cup\{i\}}\right)-v^{\mathcal{C}}\left(\mathcal{C}_{S}\right) .
\end{aligned}
$$

Combining the two last expressions, we conclude

$$
v^{\mathcal{C}}\left(\mathcal{C}_{S \cup\{i\}}\right)-v^{\mathcal{C}}\left(\mathcal{C}_{S}\right) \leq v^{\mathcal{C}}\left(\mathcal{C}_{S \cup\{i\}} \cup \mathcal{C}_{T}\right)-v^{\mathcal{C}}\left(\mathcal{C}_{T}\right) \leq v^{\mathcal{C}}\left(\mathcal{C}_{T \cup\{i\}}\right)-v^{\mathcal{C}}\left(\mathcal{C}_{T}\right) .
$$

A study about the convexity between the original game and the conference game can be found in [1], where subclasses of union stable families for which the convexity of the original game is inherited by the restricted game and the conference game respectively are given.

Notice that by means of properties of the conference game, it is possible to deduce conditions under which the Myerson value is in the core of the restricted game. It emphasizes the connection between the conference game and the Myerson value.

Theorem 4 and the fact that $\Phi(N, v) \in C(v)$ if $v$ is convex, immediately yield the following corollary.

Corollary 1 Let $(N, v, \mathcal{F})$ be a union stable cooperation structure. If the conference game $\left(\mathcal{C}, v^{\mathcal{C}}\right)$ is non-negative and convex then

$$
\mu(N, v, \mathcal{F}) \in C\left(v^{\mathcal{F}}\right) .
$$


The transmission of the convexity from the original game to the conference game requires conditions on the union stable cooperation structure. In fact, if $(N, v, \mathcal{F}) \in U S I^{N}$ and $(N, v)$ is convex then $\left(\mathcal{C}, v^{\mathcal{C}}\right)$ is convex (see $[1])$. Moreover, notice that if $(N, v)$ is superadditive and zero-normalized then $\left(\mathcal{C}, v^{\mathcal{C}}\right)$ is positive. Therefore, applying the above corollary, we can assure that on the subclass $U S I^{N}$, if the original game is convex then the Myerson value is in the core of the restricted game.

Corollary 2 Let $(N, v, \mathcal{F}) \in U S I^{N}$. If the original game $(N, v)$ is convex then

$$
\mu(N, v, \mathcal{F}) \in C\left(v^{\mathcal{F}}\right) .
$$

\section{The Myerson value and superfluous supports}

The Myerson value and the position value are allocation rules satisfying component efficiency and component dummy. In [2] the classical axiomatization of the Myerson value is given. In this section we focus on the superfluous support property which is satisfied by the Myerson value and the position value.

We first characterize the Myerson value on the subclass $U S I^{N}$ of union stable cooperation structures where the position value was characterized (see [1]) using the superfluous support property. After that, the introduction of a stronger property than the superfluous player property and superfluous support property allows us to provide new characterizations for the Myerson value on the class of all union stable cooperation structures. These axiomatizations generalize the given ones in communication situations by van den Nouweland [18].

\subsection{Axiomatizations of the Myerson value on $U S I^{N}$ using the superfluous support property}

First, we recall some standard axioms. An allocation rule $\gamma: U S^{N} \rightarrow \mathbb{R}^{N}$ is called component-efficient if for all $(N, v, \mathcal{F}) \in U S^{N}, M \in C_{\mathcal{F}}(N)$, we have $\sum_{i \in M} \gamma_{i}(N, v, \mathcal{F})=v(M)$.

An allocation rule $\gamma: U S^{N} \rightarrow \mathbb{R}^{N}$ is called component dummy if for all $i \notin \bigcup_{M \in C_{\mathcal{F}}(N)} M$, we have $\gamma_{i}(N, v, \mathcal{F})=0$.

An allocation rule $\gamma: U S^{N} \rightarrow \mathbb{R}^{N}$ is additive if

$$
\gamma(N, v+w, \mathcal{F})=\gamma(N, v, \mathcal{F})+\gamma(N, w, \mathcal{F})
$$

for all $(N, v, \mathcal{F}),(N, w, \mathcal{F}) \in U S^{N}$.

The Myerson value satisfying these three properties is shown in Algaba et al. [2]. Next we give formally the concept of superfluous support property which is defined using the conference game that was studied in the previous section.

The support $H \in \mathcal{C}$ is called superfluous for $(N, v, \mathcal{F}) \in U S^{N}$ if $v^{\mathcal{C}}(\mathcal{A})=$ $v^{\mathcal{C}}(\mathcal{A} \backslash\{H\})$, for all $\mathcal{A} \subseteq \mathcal{C}$, i.e., if support $H$ is a null player in the conference 
game. An allocation rule $\gamma: U S^{N} \rightarrow \mathbb{R}^{N}$ has the superfluous support property if $\gamma(N, v, \mathcal{F})=\gamma(N, v, \overline{\mathcal{B} \backslash\{H\}})$, for all $(N, v, \mathcal{F}) \in U S^{N}$ and for every superfluous support $H \in \mathcal{C}$ for $(N, v, \mathcal{F})$.

Theorem 5 The Myerson value $\mu: U S^{N} \rightarrow \mathbb{R}^{n}$ satisfies the superfluous support property.

Proof. Let $H \in \mathcal{C}$ be a superfluous support. We have to prove that

$$
\mu(N, v, \mathcal{F})=\mu(N, v, \overline{\mathcal{B} \backslash\{H\}}) .
$$

As

$$
\mu_{i}(N, v, \mathcal{F})=\sum_{\{S \subseteq N: i \in S\}} \eta(S)\left[v^{\mathcal{F}}(S)-v^{\mathcal{F}}(S \backslash\{i\})\right]
$$

and

$$
\mu_{i}(N, v, \overline{\mathcal{B} \backslash\{H\}})=\sum_{\{S \subseteq N: i \in S\}} \eta(S)\left[v^{\overline{\mathcal{B} \backslash\{H\}}}(S)-v^{\overline{\mathcal{B} \backslash\{H\}}}(S \backslash\{i\})\right],
$$

it is sufficient to prove that $v^{\mathcal{F}}(S)=v^{\overline{\mathcal{B} \backslash\{H\}}}(S)$, for all $S \subseteq N$.

Theorem 1 implies that $v^{\mathcal{C}}\left(\mathcal{C}_{S}\right)=v^{\mathcal{F}}(S)$.

Let $\mathcal{F}^{\prime}=\{F \in \overline{\mathcal{B} \backslash\{H\}}: F \subseteq S\}$, and let $\mathcal{B}_{S}^{\prime}$ be the basis of $\mathcal{F}_{S}^{\prime}$. Then we have that $\mathcal{B}_{S}^{\prime}=\mathcal{B}_{S} \backslash\{H\}, \mathcal{C}_{S}^{\prime} \subseteq \mathcal{C}$ and

$$
v^{\mathcal{C}}\left(\mathcal{C}_{S}^{\prime}\right)=v^{\overline{\mathcal{C}_{S}^{\prime}}}(N)=v^{\mathcal{F}_{S}^{\prime}}(N)=v^{\overline{\mathcal{B} \backslash\{H\}}}(S) .
$$

The fact that $\mathcal{B}_{S}^{\prime}=\mathcal{B}_{S} \backslash\{H\}$ and $H \in \mathcal{C}$ is a superfluous support imply

$$
v^{\mathcal{F}}(S)=v^{\mathcal{C}}\left(\mathcal{C}_{S}\right)=v^{\mathcal{C}}\left(\mathcal{C}_{S} \backslash\{H\}\right)=v^{\mathcal{C}}\left(\mathcal{C}_{S}^{\prime}\right)=v^{\overline{\mathcal{B} \backslash\{H\}}}(S) .
$$

Notice that the superfluous support property is focussed on the conference game. Whereas the conference game concentrates on the role of the supports, the restricted game does on the role of the players. However, we should take into account that the conference game is closely related to the restricted game as we have shown in Theorem 1 and the analysis of the inheritance of properties between these games carried out in Section 2. In fact, the superfluous support property will allow for a new characterization of the Myerson value.

The Myerson value also satisfies point anonymity as is shown in [2]. A union stable structure $(N, v, \mathcal{F})$ is called point anonymous if there exists a function $f:\{0,1, \ldots,|D(\mathcal{F})|\} \rightarrow \mathbb{R}$ such that $v^{\mathcal{F}}(S)=f(|S \cap D(\mathcal{F})|)$ for all $S \subseteq N$, where $D(\mathcal{F})=\left\{i \in N: \mathcal{C}_{i} \neq \emptyset\right\}$. When there is no confusion we will often write $D$ instead of $D(\mathcal{F})$.

An allocation rule $\gamma$ satisfies point anonymity if for all point anonymous $(N, v, \mathcal{F})$, there exists $\alpha \in \mathbb{R}$ such that

$$
\gamma_{i}(N, v, \mathcal{F})= \begin{cases}\alpha, & \text { for all } i \in D \\ 0, & \text { otherwise }\end{cases}
$$


Weakening point anonymity in a similar way as the corresponding property for communication structures of Borm et al. [7] is weakened by van den Brink et al. [10] yields point unanimity. A union stable structure $(N, v, \mathcal{F})$ is called point unanimous if it is point anonymous with function $f:\{0,1, \ldots,|D|\} \rightarrow \mathbb{R}$ such that $f(k)=0$, for all $k \in\{0,1, \ldots,|D|-1\}$, i.e., the restricted game is a multiple of the unanimity game on $D$.

An allocation rule $\gamma$ satisfies point unanimity if for all point unanimous $(N, v, \mathcal{F})$, there exists $\alpha \in \mathbb{R}$ such that

$$
\gamma_{i}(N, v, \mathcal{F})= \begin{cases}\alpha, & \text { for all } i \in D \\ 0, & \text { otherwise }\end{cases}
$$

Since the Myerson value satisfies point anonymity, it also satisfies the weaker point unanimity. Next we show that the above properties characterize the Myerson value when we restrict ourselves to the class $U S I^{N}$, where on the class $U S I^{N}$ the axioms are defined in a similar way as before on the full class $U S^{N}$.

Theorem 6 The Myerson value $\mu: U S I^{N} \rightarrow \mathbb{R}^{n}$ is the unique allocation rule on $U S I^{N}$ that satisfies component efficiency, component-dummy, additivity, the superfluous support property and point unanimity.

Proof. The Myerson value satisfying component efficiency, component-dummy, additivity and point anonymity (and therefore also point unanimity) is already shown in Algaba et al. [2], while the superfluous support property follows from Theorem 5 .

To show uniqueness, let $(N, v, \mathcal{F}) \in U S I^{N}$ and let $\gamma: U S I^{N} \rightarrow \mathbb{R}^{n}$ satisfy the above properties. Since the game $v$ is zero-normalized, it can be expressed as

$$
v=\sum_{\{T:|T| \geq 2\}} \beta_{T} u_{T}, \text { with } u_{T} \text { the unanimity games. }
$$

As $\gamma$ is additive, it is sufficient to show that $\gamma\left(N, \beta u_{T}, \mathcal{F}\right), \beta \in \mathbb{R}$, is unique for all $T \subseteq N$ with $|T| \geq 2$. To prove this, fix $T \subseteq N$ with $|T| \geq 2$. We distinguish two cases

Case 1. There exist no coalition $S \in \mathcal{F}$ such that $T \subseteq S$.

Since $\left(\beta u_{T}\right)^{\mathcal{F}}=\theta$, the triple $\left(N, \beta u_{T}, \mathcal{F}\right)$ is point unanimous, (because there exists $\dot{f}:\{0,1, \ldots,|D|\} \rightarrow \mathbb{R}, f(0)=\cdots=f(|D|)=0$, such that $\left(\beta u_{T}\right)^{\mathcal{F}}(H)=f(|H \cap D|)=0$, for all $\left.H \subseteq N\right)$, there exists $\alpha \in \mathbb{R}$ such that

$$
\gamma_{i}\left(N, \beta u_{T}, \mathcal{F}\right)= \begin{cases}\alpha, & \text { if } i \in D \\ 0, & \text { otherwise }\end{cases}
$$

Applying component efficiency and adding over components, we have

$$
\sum_{i \in N} \gamma_{i}\left(N, \beta u_{T}, \mathcal{F}\right)=|D| \alpha=\left(\beta u_{T}\right)^{\mathcal{F}}(N)=0 .
$$


If $D \neq \emptyset$, we have that $\alpha=0$, and therefore

$$
\gamma_{i}\left(N, \beta u_{T}, \mathcal{F}\right)=0 \text { for all } i \in N .
$$

If $D=\emptyset$, then for each $i \in N$, we have two possibilities.

If $\{i\} \notin \mathcal{F}$ then $i \notin \bigcup_{M \in C_{\mathcal{F}}(N)} M$ and $\gamma_{i}\left(N, \beta u_{T}, \mathcal{F}\right)=0$, by component dummy.

If $\{i\} \in \mathcal{F}$ then $\{i\} \in C_{\mathcal{F}}(N)$ and, applying component-efficiency

$$
\gamma_{i}\left(N, \beta u_{T}, \mathcal{F}\right)=\left(\beta u_{T}\right)^{\mathcal{F}}(\{i\})=0,
$$

since $|T| \geq 2$. In any case, $\gamma_{i}\left(N, \beta u_{T}, \mathcal{F}\right)=0$, for all $i \in N$.

Case 2. There exists a coalition $S \in \mathcal{F}$ such that $T \subseteq S$.

Consider the set $\{F \in \mathcal{F}: T \subseteq F\}$, which is non-empty since there exists $S \in \mathcal{F}$ such that $T \subseteq S$, and let

$$
\bar{T}=\bigcap\{F \in \mathcal{F}: T \subseteq F\} .
$$

The set $\bar{T}$ is non-empty and it is the minimal feasible set that contains $T$. Hence,

$$
\left(\beta u_{T}\right)^{\mathcal{F}}(H)=\beta u_{\bar{T}}(H)=\left\{\begin{array}{l}
\beta, \text { if } \bar{T} \subseteq H \\
0, \text { otherwise }
\end{array}\right.
$$

Since

(i)

$$
\left(\beta u_{T}\right)^{\mathcal{C}}(\mathcal{A})=\sum_{M \in C_{\overline{\mathcal{A}}}(N)}\left(\beta u_{T}\right)(M)=\sum_{M \in C_{\overline{\mathcal{A}}}(N)} \beta u_{T}(M)
$$

(ii) $\sum_{M \in C_{\overline{\mathcal{A}}}(N)} \beta u_{T}(M)=\beta$ if and only if $u_{T}(M)=1$, for some $M \in$ $C_{\overline{\mathcal{A}}}(N)$,

$$
u_{T}(M)=1 \Longleftrightarrow T \subseteq M, \text { with } M \in \overline{\mathcal{A}} \subseteq \mathcal{F},
$$

(iv) $\bar{T} \in \mathcal{F}$ is the smallest feasible set that contains $T$, we have

$$
u_{T}(M)=1 \Longleftrightarrow T \subseteq \bar{T} \subseteq M, \text { with } M \in \overline{\mathcal{A}} \subseteq \mathcal{F},
$$

it follows that the conference game associated to $\beta u_{T}$ is,

$$
\left(\beta u_{T}\right)^{\mathcal{C}}: 2^{\mathcal{C}} \rightarrow \mathbb{R},\left(\beta u_{T}\right)^{\mathcal{C}}(\mathcal{A})= \begin{cases}\beta, & \text { if } \bar{T} \in \overline{\mathcal{A}} \\ 0, & \text { otherwise }\end{cases}
$$

On the other hand, as $\bar{T}=\bigcup_{k \in K} B_{k}$, with $B_{k} \in \mathcal{C}$, for all $k \in K$, we have

$$
\bar{T} \subseteq M, M \in \overline{\mathcal{A}} \subseteq \mathcal{F} \Longleftrightarrow\left\{B_{k}\right\}_{k \in K} \subseteq \mathcal{A},
$$


because the expression of each non-unitary feasible coalition as a union of non-unitary supports is unique. Hence,

$$
\left(\beta u_{T}\right)^{\mathcal{C}}(\mathcal{A})=\left\{\begin{array}{l}
\beta, \text { if }\left\{B_{k}\right\}_{k \in K} \subseteq \mathcal{A}, \\
0, \text { otherwise }
\end{array}\right.
$$

All supports $B \in \mathcal{C}$ such that $B \notin\left\{B_{k}\right\}_{k \in K}$, are superfluous for the conference game. Therefore, repeatedly applying the superfluous support property to the allocation rule $\gamma$, we find

$$
\gamma\left(N, \beta u_{T}, \mathcal{F}\right)=\cdots=\gamma\left(N, \beta u_{T}, \mathcal{F}^{\prime}\right)
$$

where $\mathcal{F}^{\prime}=\left(\left\{\overline{B_{k}}\right\}_{k \in K}\right) \cup(\{\{j\}:\{j\} \in \mathcal{F}\})$.

As $\left(\beta u_{T}\right)^{\mathcal{F}^{\prime}}=\beta u_{\bar{T}}$, we have $\beta u_{\bar{T}}(S)=\beta u_{\bar{T}}(S \cap \bar{T})$ for all $S \subseteq N$, and as $\bar{T} \cap S \subseteq \bar{T}$

$$
\beta u_{\bar{T}}(S)=\beta u_{\bar{T}}(S \cap \bar{T})= \begin{cases}\beta, & \text { if } S \cap \bar{T}=\bar{T} \\ 0, & \text { otherwise. }\end{cases}
$$

Therefore, the game $\left(\beta u_{T}\right)^{\mathcal{F}^{\prime}}$ is point unanimous with

$$
D=\left\{i \in N:\left|\mathcal{C}_{i}\right|>0\right\}=\bar{T},
$$

and $f(0)=\cdots=f(|D|-1)=0, f(|D|)=\beta$.

As $\gamma$ satisfies point unanimity, we find that for all $i \in N$, there exists $\delta \in \mathbb{R}$ such that

$$
\gamma_{i}\left(N, \beta u_{T}, \mathcal{F}^{\prime}\right)= \begin{cases}\delta, & \text { if } i \in \bar{T} \\ 0, & \text { otherwise }\end{cases}
$$

Taking into account component-efficiency and component-dummy,

$$
\sum_{i \in N} \gamma_{i}\left(N, \beta u_{T}, \mathcal{F}^{\prime}\right)=|\bar{T}| \delta=\beta,
$$

and hence $\delta=\frac{\beta}{|\bar{T}|}$. Therefore, for all $i \in N$,

$$
\gamma_{i}\left(N, \beta u_{T}, \mathcal{F}^{\prime}\right)= \begin{cases}\frac{\beta}{|\bar{T}|}, & \text { if } i \in \bar{T} \\ 0, & \text { otherwise }\end{cases}
$$

Since point anonymity implies point unanimity, the Myerson value $\mu$ : $U S I^{N} \rightarrow \mathbb{R}^{n}$ is the unique allocation rule on $U S I^{N}$ that satisfies component efficiency, component-dummy, additivity, the superfluous support property and point anonymity. 


\subsection{Axiomatizations of the Myerson value on $U S^{N}$ using the strong superfluous support property}

Replacing in Theorem 6 the superfluous support property for the strong superfluous support property in the above axiomatic characterization, we provide a new characterization of the Myerson value on $U S^{N}$.

An allocation rule $\gamma: U S^{N} \rightarrow \mathbb{R}^{n}$ satisfies the strong superfluous support property if for all $(N, v, \mathcal{F}) \in U S^{N}$ and for all non-unitary supports $H \in \mathcal{C}$ whose absence does not influence the $\mathcal{F}$-restricted game, i.e.,

$$
v^{\mathcal{F}}(S)=v^{\overline{\mathcal{B} \backslash\{H\}}}(S), \text { for all } S \subseteq N,
$$

it is verified that

$$
\gamma(N, v, \mathcal{F})=\gamma(N, v, \overline{\mathcal{B} \backslash\{H\}}) .
$$

Notice that the strong superfluous support property is centered on the restricted game instead of the conference game. This new property states that the solution is the same after removing a support which has no influence on the communication in the sense that it does not change the worth of coalitions in the restricted game.

Also notice that the strong superfluous support property implies the superfluous support property but the reverse is not true. Indeed, from the proof of Theorem 5 it follows that if $H \in \mathcal{C}$ is a superfluous support then $v^{\mathcal{F}}(S)=v^{\overline{\mathcal{B} \backslash\{H\}}}(S)$, for all $S \subseteq N$. Therefore, if an allocation rule satisfies the strong superfluous support property then

$$
\gamma(N, v, \mathcal{F})=\gamma(N, v, \overline{\mathcal{B} \backslash\{H\}}) .
$$

However, if $H \in \mathcal{C}$ satisfies that $v^{\mathcal{F}}(S)=v^{\overline{\mathcal{B} \backslash\{H\}}}(S)$, for all $S \subseteq N$ then the support $H$ is not necessarily a superfluous support.

Before establishing the axiomatic characterization of the Myerson value on the class of all union stable structures, we prove an interesting result which assures that for all $(N, v, \mathcal{F}) \in U S^{N}$ the outcome in the game is the same as the restricted game for any allocation rule satisfying component efficiency, component-dummy, additivity and point unanimity.

Lemma 1 If $\gamma$ is an allocation rule that satisfies additivity, component efficiency, component-dummy and point unanimity, then

$$
\gamma(N, v, \mathcal{F})=\gamma\left(N, v^{\mathcal{F}}, \mathcal{F}\right)
$$

for all $(N, v, \mathcal{F}) \in U S^{N}$.

Proof. By additivity of $\gamma$, it suffices to show that $\gamma\left(N, v-v^{\mathcal{F}}, \mathcal{F}\right)=0$, for all $(N, v, \mathcal{F}) \in U S^{N}$. First $\left(N, v-v^{\mathcal{F}}, \mathcal{F}\right)$ is point unanimous since $\left(v-v^{\mathcal{F}}\right)^{\mathcal{F}}(S)=0$, for all $S \subseteq N$. (Indeed, let $f:\{0,1, \ldots,|D|\} \rightarrow \mathbb{R}$ such 
that $f(k)=0$, with $k=0,1, \ldots,|D|$. Then, $\left(v-v^{\mathcal{F}}\right)^{\mathcal{F}}(S)=f(|S \cap D|)=0$, for all $S \subseteq N$.) Therefore, there exists $\alpha \in \mathbb{R}$ such that

$$
\gamma_{i}\left(N, v-v^{\mathcal{F}}, \mathcal{F}\right)= \begin{cases}\alpha, & \text { if } i \in D \\ 0, & \text { otherwise }\end{cases}
$$

Let $M \in C_{\mathcal{F}}(N)$ such that $|M|>1$. As $\gamma$ satisfies component efficiency, we have that

$$
\sum_{i \in M} \gamma_{i}\left(N, v-v^{\mathcal{F}}, \mathcal{F}\right)=\alpha|M|=\left(v-v^{\mathcal{F}}\right)(M)=0
$$

and thus $\alpha=0$. Hence, $\gamma_{i}\left(N, v-v^{\mathcal{F}}, \mathcal{F}\right)=0$, for all $i \in N$.

Obviously, the above lemma is satisfied when substituting point unanimity for point anonymity.

Next, as mentioned above, we establish a characterization of the Myerson value on $U S^{N}$ only by replacing in the given characterization the superfluous support property with the strong superfluous support property. Here, we use the characterization given in [2], where the Myerson value is characterized using the superfluous player property, which is analogous to the superfluous support property changing the conference game for the restricted game and the role of the supports for players.

Let $(N, v, \mathcal{F}) \in U S^{N}$. A player $i \in N$ is called superfluous for $(N, v, \mathcal{F})$ if $v^{\mathcal{F}}(S)=v^{\mathcal{F}}(S \backslash\{i\})$, for all $S \subseteq N$. An allocation rule $\gamma$ satisfies the $s u$ perfluous player property if for all $(N, v, \mathcal{F})$ and every player $i \in N$ that is superfluous for $(N, v, \mathcal{F})$ it holds $\gamma(N, v, \mathcal{F})=\gamma\left(N, v, \mathcal{F}_{N \backslash\{i\}}\right)$, where $\mathcal{F}_{N \backslash\{i\}}=\{F \in \mathcal{F}: F \subseteq N \backslash\{i\}\}$.

Theorem 7 The Myerson value $\mu: U S^{N} \rightarrow \mathbb{R}^{n}$ is the unique allocation rule on $U S^{N}$ that satisfies component efficiency, component-dummy, additivity, the strong superfluous support property and point unanimity.

Proof. By definition, it is straightforward that the Myerson value satisfies the strong superfluous support property. Therefore, it only remains to prove uniqueness. Let $\gamma$ be an allocation rule on $U S^{N}$ that satisfies component efficiency, component-dummy, additivity, the strong superfluous support property and point unanimity. To prove that $\gamma$ is uniquely determined, we first prove that if an allocation rule on $U S^{N}$ satisfies the strong superfluous support property then it satisfies the superfluous player property, and hence as the Myerson value is the unique allocation rule on $U S^{N}$ that satisfies additivity, the superfluous player property and point unanimity [2, Theorem 3.7] we deduce that $\mu=\gamma$. (In concrete, this characterization was shown with point anonymity, but it is easy to check that the result holds with point unanimity).

Let $i \in N$ be a superfluous player for $(N, v, \mathcal{F})$, i.e.,

$$
v^{\mathcal{F}}(S)=v^{\mathcal{F}}(S \backslash\{i\}) \text {, for all } S \subseteq N .
$$


We have to prove that

$$
\gamma(N, v, \mathcal{F})=\gamma\left(N, v, \mathcal{F}_{N \backslash\{i\}}\right),
$$

where $\mathcal{F}_{N \backslash\{i\}}=\{F \in \mathcal{F}: F \subseteq N \backslash\{i\}\}=\{F \in \mathcal{F}: F \subseteq N, i \notin F\}=\overline{\mathcal{B} \backslash \mathcal{B}_{i}}$ with $\mathcal{B}_{i}=\{B \in \mathcal{B}: i \in B\}$.

As $\gamma$ is an additive allocation rule that satisfies component efficiency, component-dummy and point unanimity, by Lemma 1 we have

$$
\gamma(N, v, \mathcal{F})=\gamma\left(N, v^{\mathcal{F}}, \mathcal{F}\right) .
$$

It is satisfied that $C_{\mathcal{F}}(S \backslash\{i\})=C_{\mathcal{F}_{N \backslash\{i\}}}(S)$, for all $S \subseteq N$. Hence, if $i \in N$ is a superfluous player, then for all $S \subseteq N$

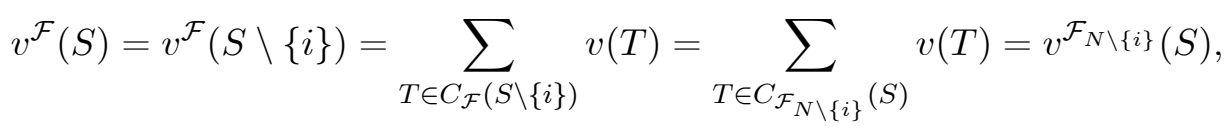

and thus, applying (3),

$$
\gamma(N, v, \mathcal{F})=\gamma\left(N, v^{\mathcal{F}}, \mathcal{F}\right)=\gamma\left(N, v^{\left.\mathcal{F}_{N \backslash\{i\}}, \mathcal{F}\right)} .\right.
$$

As $\mathcal{F}_{N \backslash\{i\}}=\overline{\mathcal{B} \backslash \mathcal{B}_{i}}$ and $v^{\mathcal{F}}=v^{\mathcal{F}_{N \backslash\{i\}}}$, then any support $B \in \mathcal{B}_{i}$ has no influence in the $\mathcal{F}$-restricted game since for all $S \subseteq N$,

$$
v^{\mathcal{F}}(S)=v^{\mathcal{F}_{N \backslash\{i\}}}(S)=v^{\overline{\mathcal{B} \backslash \mathcal{B}_{i} \backslash\{B\}}}(S),
$$

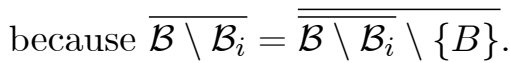

Applying the strong superfluous support property repeatedly, for all $B \in$ $\mathcal{B}_{i}$, we have

$$
\gamma(N, v, \mathcal{F})=\gamma\left(N, v^{\mathcal{F}_{N \backslash\{i\}}}, \mathcal{F}\right)=\gamma\left(N, v^{\left.\mathcal{F}_{N \backslash\{i\}}, \mathcal{F}_{N \backslash\{i\}}\right)}=\gamma\left(N, v, \mathcal{F}_{N \backslash\{i\}}\right) .\right.
$$

Therefore, $\gamma$ is an allocation rule that satisfies the superfluous player property and we conclude

$$
\gamma(N, v, \mathcal{F})=\mu(N, v, \mathcal{F}), \text { for all }(N, v, \mathcal{F}) \in U S^{N} .
$$

Taking into account that point anonymity implies point unanimity, the Myerson value $\mu: U S^{N} \rightarrow \mathbb{R}^{n}$ is the unique allocation rule on $U S^{N}$ that satisfies component efficiency, component-dummy, additivity, the strong superfluous support property and point anonymity.

\section{Conclusions}

This paper makes some contributions to cooperative game theory with restricted cooperation in a framework that not only allows for the unification and generalization of different research lines such as communication situations or permission structures but also the analysis of economic applications which 
arise in this context. At first sight, the Myerson value and the conference game, and therefore the supports, are not related. In this paper, we show the existence of a direct relationship between the Myerson value and the conference game. First, a study between the restricted game and the conference game as well as an analysis of the transmission of properties reveals the close connection between the Myerson value and the conference game. In fact, by means of the conference game it can be established when the Myerson value is in the core of the restricted game, without requirements about the structure or the restricted game. Second, an analysis about superfluous supports, or null players in the conference game, and the Myerson value leads us to characterizations of the Myerson value on a special subclass of union stable systems that contains those coming from cycle-free graphs. Finally, strengthening the superfluous support property and superfluous player property, two new characterizations for the Myerson value are provided on the class of all union stable structures. Therefore, important properties and characterizations of the Myerson value for games on union stable systems can be stated by the study of the conference game.

For communication situations, van den Brink et al. [10] introduce the class of Harsanyi power solution which can be obtained by distributing the Harsanyi dividends in the point game proportional to some power measure for communication graphs. This class contains the Myerson value which is obtained by using the equal power measure. This approach is generalized to games on union stable systems by Algaba et al. [5]. Although every Harsanyi power solution satisfies the superfluous support property on the class $U S I^{N}$, in general they do not satisfy this property on $U S^{N}$, and therefore the same is true for the strong superfluous support property. Thus, in some sense the superfluous support property and the role of the conference game are characteristic for the Myerson value within the class of Harsanyi power solutions. On the other hand, the position value, which has a quite different interpretation and definition than the Myerson value (introduced through the conference game instead of the restricted game), also satisfies the superfluous support property on $U S^{N}$. However, this is not a Harsanyi power solution, although it is on the class $U S I^{N}$.

\section{References}

[1] Algaba E, Bilbao JM, Borm P, López JJ (2000) The position value for union stable systems. Math. Meth. Oper. Res. 52:221-236

[2] Algaba E, Bilbao JM, Borm P, López JJ (2001) The Myerson value for union stable structures. Math. Meth. Oper. Res. 54:359-371

[3] Algaba E, Bilbao JM, López JJ (2001) A unified approach to restricted games. Theory and Decision 50:333-345

[4] Algaba E, Bilbao JM, López JJ (2004) The position value in communication structures. Math Meth Oper Res 59:465- 477

[5] Algaba E, Bilbao JM, Brink R van den (2011) Harsanyi power solutions for games on union stable systems . Mimeo 
[6] Bondareva O (1963) Some applications of linear programming methods to the theory of cooperative games. Problem Kibernet 10:119-139

[7] Borm P, Owen G, Tijs SH (1992) On the position value for communication situations. SIAM J. Discrete Math. 5:305-320

[8] Brink $\mathrm{R}$ van den (1997) An axiomatization of the disjunctive permission value for games with a permission structure. Int. J. Game Theory 26:2743

[9] Brink R van den (2011) On hierarchies and communication. Forthcoming in Soc Choice Welf.

[10] Brink R van den, Laan G van der, Pruzhansky V (2011) Harsanyi power solutions for graph-restricted games. Int. J. Game Theory 40:87-110.

[11] Faigle U, Grabish M, Heyne M (2010) Monge extensions of cooperation and communication structures. European J. of Operational Research 206:104-110

[12] Gilles RP, Owen G, Brink R van den (1992) Games with permission structures: the conjunctive approach. Int. J. Game Theory 20:277-293

[13] Meessen R (1988) Communication games. Master's thesis, Dept of Mathematics, University of Nijmegen, the Netherlands. (In Dutch)

[14] Myerson RB (1977) Graphs and cooperation in games. Math. Oper. Res. $2: 225-229$

[15] Myerson RB (1980) Conference structures and fair allocation rules. Int. J. Game Theory 9:169-182

[16] Nouweland A van den, Borm P (1990) On the convexity of communication games. Int. J. Game Theory 19: 421-430

[17] Nouweland A van den, Borm P, Tijs SH (1992) Allocation rules for hypergraph communication situations. Int. J. Game Theory 20:255-268

[18] Nouweland A van den (1993) Games and graphs in economics situations. PhD Dissertation, Tilburg University, The Netherlands

[19] Owen G (1986) Values of graph-restricted games. SIAM J. on Algebraic and Discrete Methods 7:210-220

[20] Potters JAM, Reijnierse H (1995) $\Gamma$-Component additive games. Int. J. Game Theory 24:49-56

[21] Shapley LS (1953) A value for $n$-person games. In: Kuhn HW, Tucker AW (eds.). Contributions to the Theory of Games, Vol II. Princeton UP, pp. 307-317

[22] Shapley LS (1967) On balanced sets and cores. Naval Research Logistics Quarterly 14:453-460 